Approved for public release; distribution is unlimited.

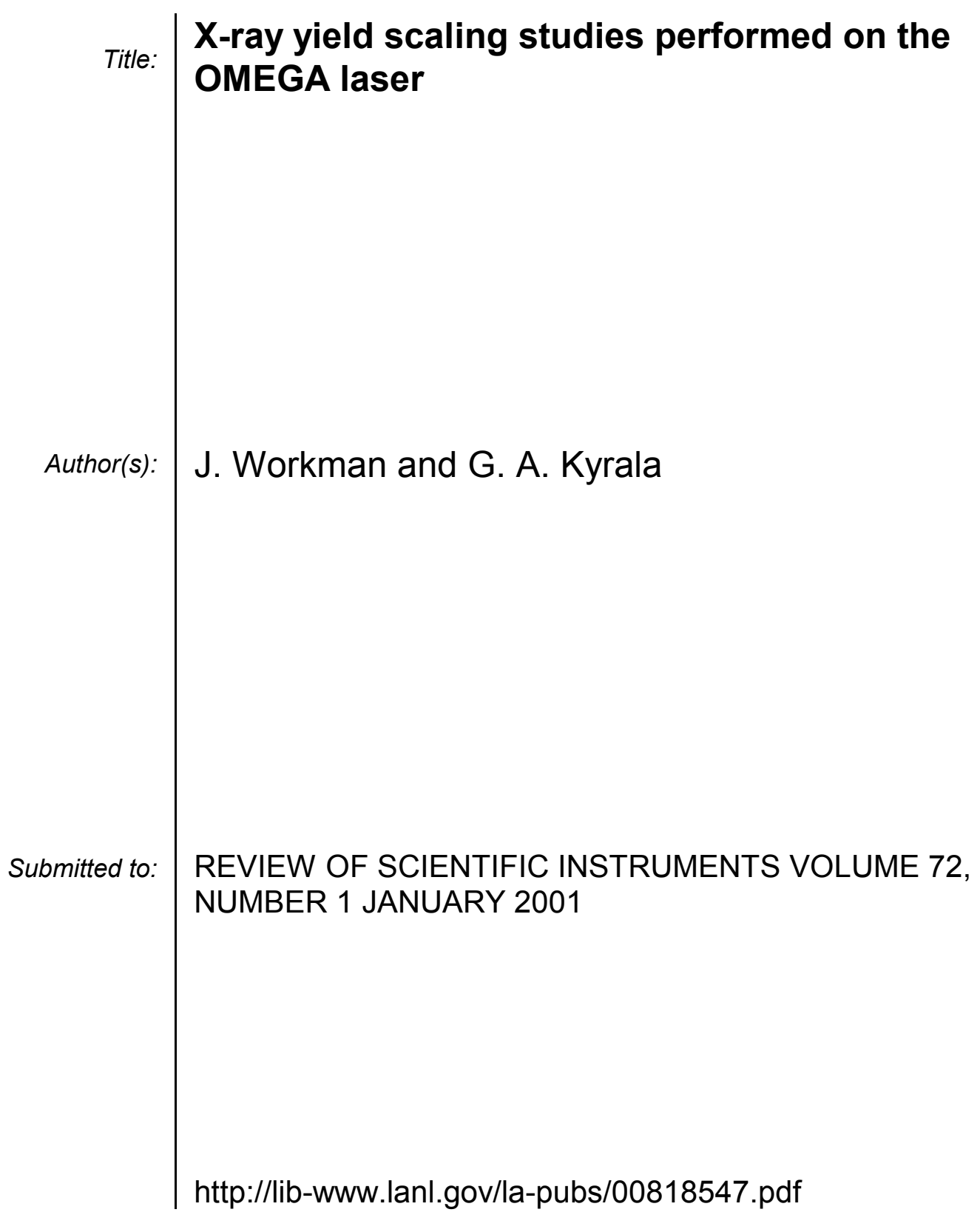

Los Alamos National Laboratory, an affirmative action/equal opportunity employer, is operated by the University of California for the U.S. Department of Energy under contract W-7405-ENG-36. By acceptance of this article, the publisher recognizes that the U.S. Government retains a nonexclusive, royaltyfree license to publish or reproduce the published form of this contribution, or to allow others to do so, for U.S. Government purposes. Los Alamos National Laboratory requests that the publisher identify this article as work performed under the auspices of the U.S. Department of Energy. Los Alamos National Laboratory strongly supports academic freedom and a researcher's right to publish; as an institution, however, the Laboratory does not endorse the viewpoint of a publication or guarantee its technical correctness. 
Los Alamos National Laboratory, an affirmative action/equal opportunity employer, is operated by the University of California for the U.S. Department of Energy under contract W-7405-ENG-36. By acceptance of this article, the publisher recognizes that the U.S. Government retains a nonexclusive, royaltyfree license to publish or reproduce the published form of this contribution, or to allow others to do so, for U.S. Government purposes. Los Alamos National Laboratory requests that the publisher identify this article as work performed under the auspices of the U.S. Department of Energy. Los Alamos National Laboratory strongly supports academic freedom and a researcher's right to publish; as an institution, however, the Laboratory does not endorse the viewpoint of a publication or guarantee its technical correctness. 


\title{
X-ray yield scaling studies performed on the OMEGA laser
}

\author{
J. Workman a) and G. A. Kyrala \\ Los Alamos National Laboratory, Box 1663, Mail Stop E-526, Los Alamos, New Mexico 87545
}

(Presented on 20 June 2000)

\begin{abstract}
We have performed experiments with planar targets on the OMEGA laser facility at the University of Rochester. These experiments investigated the scaling of x-ray yield and conversion efficiency with the laser energy and focusing properties for several different target materials. The experiments were also designed to investigate the feasibility of high-energy backlighters under typical irradiance geometries. The scaling of $\mathrm{Fe}$ emission near $6.7 \mathrm{keV}$ was investigated by varying laser irradiance from $10^{14}$ to $10^{16} \mathrm{~W} / \mathrm{cm}^{2}$. In addition, the scaling of $\mathrm{x}$-ray yield with emitted $\mathrm{x}$-ray energy was studied at fixed laser irradiance near $10^{16} \mathrm{~W} / \mathrm{cm}^{2}$ for $\mathrm{Fe}, \mathrm{Zn}$, and Ge. The time-integrated spectra as well as filtered x-ray film gave relative x-ray yields. (c) 2001 American Institute of Physics.
\end{abstract}

[DOI: $10.1063 / 1.1318251]$

\section{INTRODUCTION}

$\mathrm{X}$-ray imaging is a standard and important technique used to determine density, symmetry, size, interface, and opacity information in high-density objects. The image can be collected either from the self-emission of the object or by backlighting from a separate $\mathrm{x}$-ray source behind the object. The objects to be imaged are typically quite small in laserbased experiments, often requiring spatial resolutions of ten microns or less. ${ }^{1}$ These small spatial resolution scales and high densities put very high demands on the properties of the $\mathrm{x}$-ray source used for backlighting.

The quality of an x-ray image is directly related to the flux of the x-ray source at the detector, the efficiency of the detector, and the $\mathrm{x}$-ray energy. Because the recorded $\mathrm{x}$-ray signal depends on the backlit object opacity, length, and $\mathrm{x}$-ray source strength, an understanding of how to produce the desired x-ray flux at the appropriate energy is crucial in the design of an experiment. Determining the appropriate laser conditions for a given $\mathrm{x}$-ray source is not easily modeled. ${ }^{2}$ It is therefore necessary to determine the scaling laws experimentally. Laser parameters controlling x-ray yield include laser irradiance, wavelength, spot size, polarization, angle of incidence, beam smoothing, and pulse duration. In addition, target material and density will determine the emitted x-ray spectral fluence and absorbed laser energy. Over the past two decades several experiments have been performed to determine $\mathrm{x}$-ray yield and conversion efficiency as a function of laser irradiance, wavelength, and pulse duration. ${ }^{3-8}$ In addition, the drastic decrease in x-ray conversion efficiency with heavier target materials has been demonstrated experimentally. ${ }^{9-11}$

The problem encountered by an experimentalist designing an x-ray backlighter source is the scarcity of data points and the confusion created by discrepancies in the available data. The discrepancies can often be explained by the differences between lasers and is manifested by differences in the x-ray spectra. ${ }^{4}$ In an attempt to extend the available data and

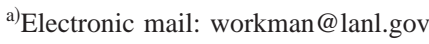

try to understand the scaling and discrepancies, we have investigated $x$-ray yield scaling on the OMEGA laser facility at the University of Rochester. ${ }^{12}$ In this article we present two measurements, the scaling of high-energy He-like x-ray yield in Fe targets as a function of laser irradiance and a comparison of yield from $\mathrm{Fe}$ (at $6.7 \mathrm{keV}$ ), $\mathrm{Zn}$ (at $9.0 \mathrm{keV}$ ), and $\mathrm{Ge}$ (at $10.3 \mathrm{keV}$ ) at the same laser irradiance.

\section{EXPERIMENT}

Targets consisted of flat round disks of 3 and $1 \mathrm{~mm}$ diameter. The 1-mm-diam targets were used when laser beams were focused tightly and overlapped. The smaller targets allowed alignment fiducials to be viewed in the so called "high-resolution alignment system," necessary for precise overlap of the tightly focused laser spots. Primary diagnostics included a time-integrated crystal spectrometer and an aluminum step-wedge filtered direct exposure film (DEF) $\mathrm{x}$-ray film pack. In addition, low-resolution streaked spectra, static pinhole images, and gated framing camera images were also recorded. Targets were irradiated with up to six beams with an angular cone $23^{\circ}$ from the target normal using a $1 \mathrm{~ns}$ temporally square profile at a wavelength of $351 \mathrm{~nm}$.

As shown in Fig. 1, targets were oriented normal to the H7-H14 axis where opposing ten-inch diagnostic manipulators (TIMs) had views normal to the target surface from front and rear. One primary diagnostic, a time-integrated spectrometer (Henway spectrometer ${ }^{13}$ ), looked at an angle of $34^{\circ}$ with respect to the normal at the irradiated side of the target. The time-integrated crystal spectrometer consisted of four channels of data using separate convex crystals. Overlap of spectral coverage on three crystals allowed a measure of relative reflectivity. Crystals in the spectrometer included, ammonium dihydrogen phosphate (ADP), pertaerthritol (PET), and Si. The x-ray spectra were recorded on DEF $\mathrm{x}$-ray film. The film was processed using the method prescribed by Henke et al. ${ }^{14}$ and then digitized using a micodensitometer with a step size of $20 \times 20 \mu \mathrm{m}^{2}$. The actual exposure on the film as a function of $x$-ray energy was then calculated from the Henke film data calibration. ${ }^{14}$ In addition 


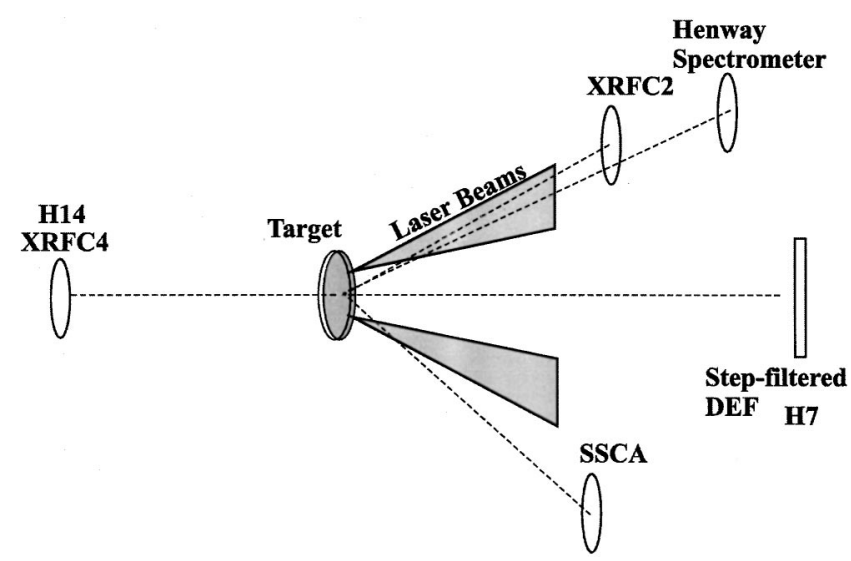

FIG. 1. Experimental setup on the OMEGA chamber. Target is positioned at the target chamber center. X-ray framing cameras are positioned normal to the rear surface and at $37^{\circ}$ from the irradiated surface at a magnification of $6 \times$. The streaked spectrometer is positioned at $79^{\circ}$ from the target normal and the time-integrated $\mathrm{x}$-ray spectrometer is positioned at $34^{\circ}$.

to the additional diagnostics shown in Fig. 1, six static pinhole cameras at magnifications of $4 \times$ viewed the target from front and rear at various angles, including a view of the rear side at an angle of $34^{\circ}$ with respect to the normal.

The laser irradiance on target was varied by changing the number of laser beams used or by changing the laser spot size. Laser spot sizes were varied from 600 down to $\sim 100$ $\mu \mathrm{m}$ diameter while overlapping beams. Up to six beams were used with a total energy of $3 \mathrm{~kJ}$. Overlapping of the beams was monitored both by the gated and static pinhole imagers.

\section{RESULTS}

A total of 12 targets were shot on OMEGA consisting mainly of Fe and Ge with one shot using a $\mathrm{Zn}$ target. The primary results are from the time-integrated $\mathrm{x}$-ray spectrometer. A discussion of x-ray yield from the step-filtered $\mathrm{x}$-ray film is incomplete at this time.

The scaling of x-ray yield with laser irradiance for Fe targets was performed over a range of $4 \times 10^{14}$ to 2 $\times 10^{16} \mathrm{~W} / \mathrm{cm}^{2}$, where irradiance is defined as total energy divided by pulse duration and laser spot size. The first three irradiances $\left(4 \times 10^{14}, 7.5 \times 10^{14}\right.$, and $\left.1 \times 10^{15} \mathrm{~W} / \mathrm{cm}^{2}\right)$ were obtained for beams defocused to a diameter of $600 \mu \mathrm{m}$ (no phase plates or spectral smoothing were used) with differing numbers of beams. The defocused beams were converging at the target surface. In order to reach higher irradiance, the laser focal diameter was decreased from 600 to $300 \mu \mathrm{m}$ and finally to $100 \mu \mathrm{m}$ giving a maximum irradiance of about 2 $\times 10^{16} \mathrm{~W} / \mathrm{cm}^{2}$. Although changing the focal spot changes the dimensionality of the problem, this was the most practical method of obtaining high irradiance. (The irradiance using tightly focused beams is of practical interest for point-backlit imaging. ${ }^{15}$ )

Figure 2 is a summary of the x-ray yield data around 6.7 $\mathrm{keV}$ obtained with the time-integrated spectrometer as a function of laser irradiance for Fe targets. Data points are integrals of film exposure over a given energy band. This band was kept small to minimize contributions from film

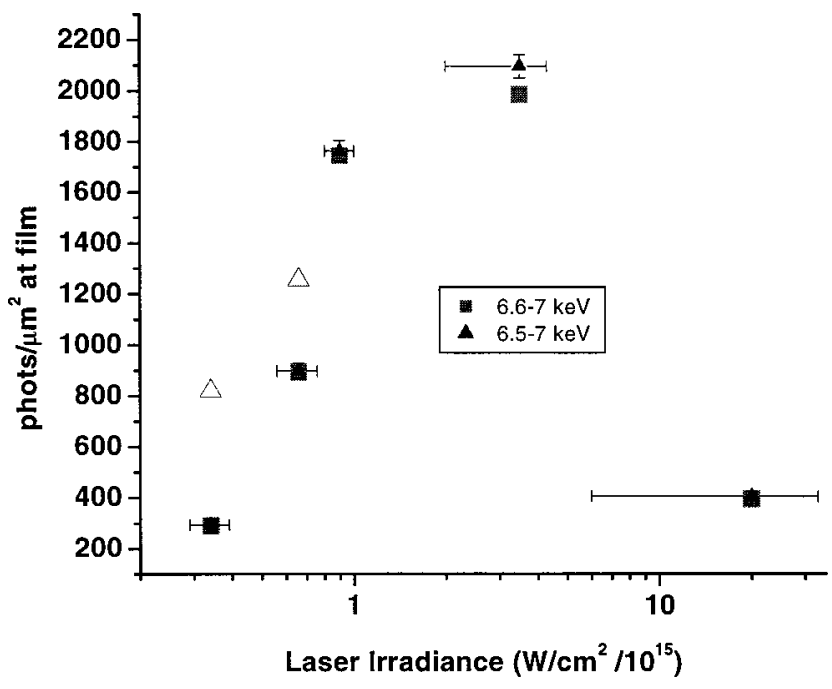

FIG. 2. X-ray yield scaling from Fe targets as a function of laser irradiance. Vertical axis is photons reaching the detector corrected for filters. The open symbols are the lower irradiance data scaled to the energy of the other points $(3 \mathrm{~kJ})$.

noise and to emulate the spectra typically selected for pinhole imaging. (The energy range in a standard pinhole imager is determined by a filter band-pass of the same material as the target placed in the path of the broadband x-ray emission. This filter is in place to monochromatize the $x$-ray energy, usually selecting the He-like transition for $Z>14$.) The vertical axis is in terms of photons reaching the DEF film plane corrected for filter transmissions. This does not include the reflectivity of the crystal, which is an unknown at this time. The three lowest irradiances were obtained for two, four, and six beams overlapped and defocused to a $600 \mu \mathrm{m}$ diameter. The data point at $4 \times 10^{15} \mathrm{~W} / \mathrm{cm}^{2}$ used six beams overlapped at a diameter of $300 \mu \mathrm{m}$. The highest irradiance data point was obtained using six beams tightly focused to a diameter of about 100-150 $\mu \mathrm{m}$. The total energy was typically $3 \mathrm{~kJ}$ when six beams were used.

The peak in the x-ray yield in Fe occurred for an irradiance of $4 \times 10^{15} \mathrm{~W} / \mathrm{cm}^{2}$ and dropped off sharply above this value. For the data points at and above $4 \times 10^{15} \mathrm{~W} / \mathrm{cm}^{2}$, there was a measurable continuum under the lines. Uncertainty in the irradiance is due primarily to the spot size and is largest with the tightly focused overlapped beams. Because the tightly focused beams may not have been perfectly overlapped, the highest irradiance data point is bracketed on the low end by overexposed static pinhole images of the x-ray source. This is very likely a large overestimate of the laser spot size but is nonetheless included pending further analysis of the gated x-ray images. The high-irradiance side of the error bar is determined by measured minimum spot sizes achievable on OMEGA and the assumption that beams were perfectly overlapped. Because the defocused beams are much easier to overlap, the error bars are significantly smaller. The open symbols included in Fig. 2 are the first two data points rescaled in energy to the successive data points. These rescaled data points along with the other points using six beams give an indication of how the conversion efficiency scales. 


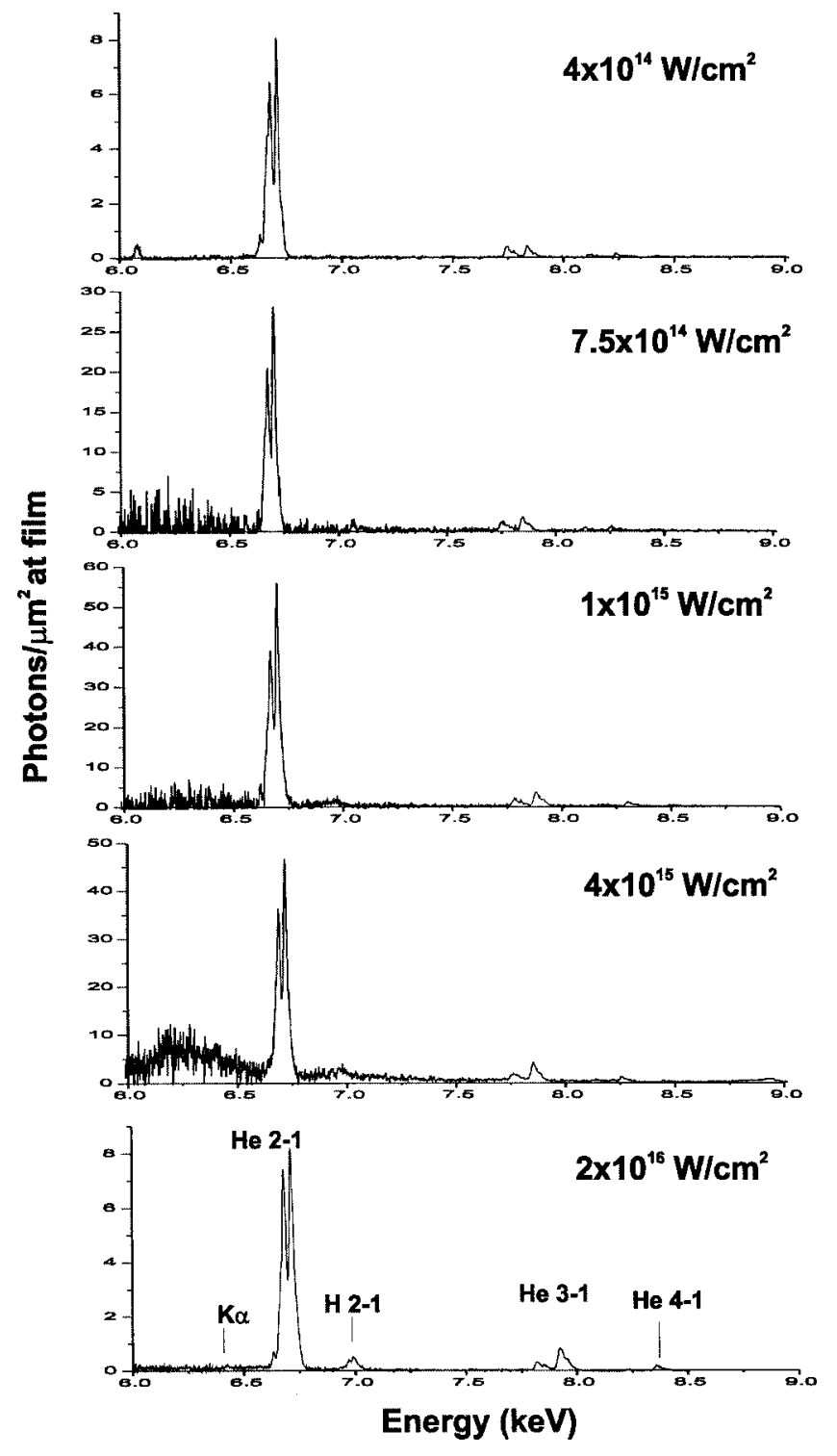

FIG. 3. Fe spectra obtained at different laser irradiances. The spectra have been post-processed to correct for filter responses ( $\mathrm{Al}$ attenuators). The vertical axis is in terms of photons per square micron recorded at the DEF film plane, corrected for filters.

The spectra from which the above Fe data were taken are shown in Fig. 3. These spectra were taken on two different PET crystals with three different attenuation channels. Aluminum was used for attenuation, as it has a relatively flat transmission over a large energy range and minimizes fluorescence effects. ${ }^{16}$ When corrected for filter transmission, the Fe He-like 2-1 transition is by far the most prominent spectral feature. The H-like 2-1 transition is only pronounced at the higher laser irradiances. He-like 3-1 and 4-1 are quite prominent in all the spectra before correction for the aluminum filters, which have a larger transmission at higher energies. There is only a small trace of $K-\alpha(6.4 \mathrm{keV})$ emission (cold material emission) at the highest irradiance. The lack of $K-\alpha$ emission is puzzling, as we found an apparent large contribution from high energy $\mathrm{x}$ rays from analysis of the step-filtered $\mathrm{x}$-ray film. The spectra, for an irradiance at and above $4 \times 10^{15} \mathrm{~W} / \mathrm{cm}^{2}$, shows the He-like 2-1 emission line sitting on top of a continuum. This background is real, con-

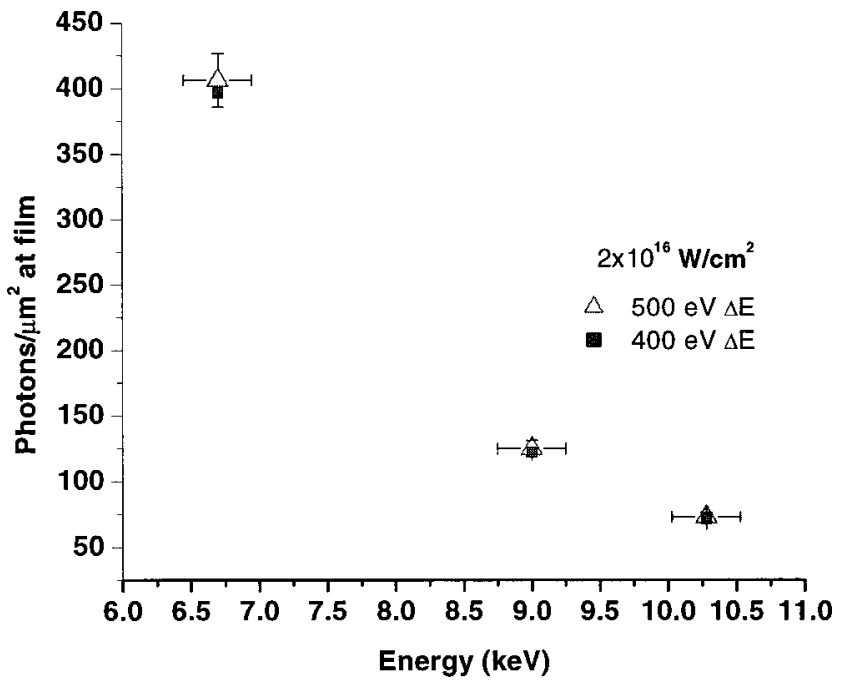

FIG. 4. X-ray yield scaling as a function of $x$-ray energy (target $Z$ ) using Fe, $\mathrm{Zn}$, and Ge targets. Laser irradiance was $2 \times 10^{16} \mathrm{~W} / \mathrm{cm}^{2}$ using six tightly focused overlapped beams. Total laser energy was $3 \mathrm{~kJ}$.

firmed by the same level of background on all three crystals, and is a large contributor to the integrated yield values in Fig. 2.

Another important scaling parameter is x-ray energy, which is associated with the atomic number, $Z$, of the target material. As target $Z$ increases, more laser energy is required in the ionization process, leaving less laser energy to be converted into the desired $x$ rays. Figure 4 shows the relative decrease in $\mathrm{x}$-ray yield for three different targets of increasing atomic number under similar irradiance conditions. The targets were irradiated at $2 \times 10^{16} \mathrm{~W} / \mathrm{cm}^{2}$ with six beams of OMEGA overlapped and tightly focused. The total energy in the six beams was $3 \mathrm{~kJ}$. The targets were Fe, $\mathrm{Zn}$, and Ge with corresponding He-like 2-1 emission at 6.7, 9.0, and 10.3 $\mathrm{keV}$, respectively. Yield is integrated over 400 and $500 \mathrm{eV}$ bandwidths, which bracket cold $K \alpha$, He-like 2-1 and H-like 2-1 emission. Because the irradiance conditions are similar, the yield scaling is identical to the conversion efficiency scaling. Both yield and conversion efficiency are measured to decrease as $(h \nu)^{-4.0}$ for this irradiance, where $h \nu$ is the $\mathrm{x}$-ray emission energy.

Figure 5 shows the time-integrated spectra corresponding to the data in Fig. 4. The Fe spectrum has been reduced by a factor of 10 in order to be on scale with the $\mathrm{Zn}$ and $\mathrm{Ge}$ data. In addition, the Fe spectrum has been corrected for a factor of 4 difference in reflectivity between the two crystals used. (This corresponds to the scale difference in Figs. 3 and 5.) The $\mathrm{Zn}$ and Ge spectra were taken using a $\mathrm{Si}(111)$ crystal while the Fe was taken using a PET crystal. The relative reflectivities of the two crystals were obtained using redundant information from the $\mathrm{Zn}$ and Fe spectrum appearing on both crystals.

Results from an aluminum step-filtered film pack showed an anomalously high transmission for the energy we expected to be measuring. A plot of measured exposure on the DEF film versus filter thickness should have resulted in a single exponential curve fitting the absorption coefficient at the energy of interest. Instead, we found a multiexponential 


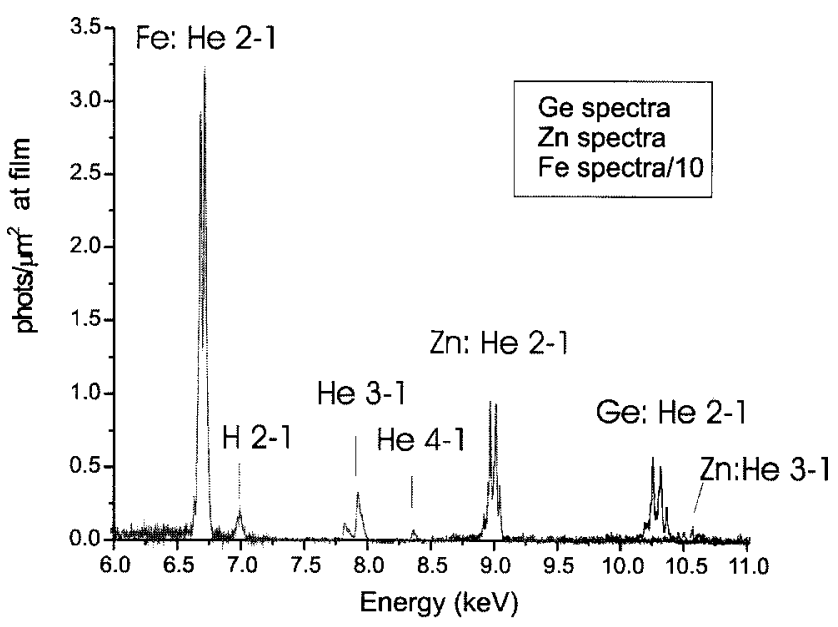

FIG. 5. Time-integrated x-ray spectra obtained from Fe, Zn, and Ge flat disk targets. $\mathrm{Zn}$ and $\mathrm{Ge}$ were recorded using a $\mathrm{Si}(111)$ crystal while the $\mathrm{Fe}$ spectra, recorded using a PET crystal, has been scaled for relative crystal reflectivity and divided by 10 . Vertical axis is the signal reaching the film corrected for filters.

decay. Initial analysis indicated that much of the signal was coming from $x$ rays with energies above $14 \mathrm{keV}$, beyond the range of the spectrometer limit. Further analysis will be presented in a future article.

\section{DISCUSSION}

The scaling in Fe of the $\mathrm{x}$-ray yield with laser irradiance and target material demonstrate some of the important concerns an experimentalist must weigh in designing an x-ray imaging system. Data has been presented primarily in terms of total photon number instead of conversion efficiency. When irradiance is varied using laser energy, $\mathrm{x}$-ray yield can continue to increase despite a decrease in conversion efficiency. The peak in yield comes about from an efficient coupling of laser energy into the desired ionization state. At very low irradiance, the plasma temperature and density are not sufficient to support the ionization state. At very high irradiance the coupling of laser energy into hot electrons and other nonthermal modes may become significant. As the target atomic number is increased, the peak in yield will require hotter plasmas (higher irradiance and more energy), supporting the higher ionization states required for He-like emission as evidenced in the decrease in yield with target $Z$.

As noted in the introduction, there are many parameters that affect $\mathrm{x}$-ray yield including target and laser properties. This experiment only begins to explore the effects of these parameters on the scaling of x-ray yield. Scaling of x-ray yield with laser irradiance included changing two important parameters, both laser energy and spot size. The size of the laser-heated region can change the energy transport properties in the target drastically. With a limited number of target shots available for diagnostic development only a small subset of scaling parameters could be investigated.

In conclusion, measurements of $x$-ray yield at $6.7 \mathrm{keV}$ in Fe targets show a peak near an irradiance of 4 $\times 10^{15} \mathrm{~W} / \mathrm{cm}^{2}$. Measurements of Fe, $\mathrm{Zn}$, and Ge X-ray yield at $6.7,9.0$, and $10.3 \mathrm{keV}$, respectively, show decreasing yield as a function of emitted x-ray energy that scales as $(h \nu)^{-4}$.

\section{ACKNOWLEDGMENTS}

The authors would like to thank the MST 7 target fabrication team, P. Walsh, S. Evans, and T. Sedillo of Los Alamos National Laboratory, V. Rekow and R. Costa of Lawrence Livermore Laboratory, and the OMEGA operations crew. This work was performed at Los Alamos National Laboratory under the auspices of the U.S. Department of Energy under Contract No. W-7405-ENG-36.

${ }^{1}$ S. R. Goldman, S. E. Caldwell, M. D. Wilke, D. C. Wilson, C. W. Barnes, W. W. Hsing, N. D. Delamater, G. T. Schappert, J. W. Grove, E. L. Lindman, J. M. Wallace, R. P. Weaver, A. M. Dunne, M. J. Edwards, P. Graham, and B. R. Thomas, Phys. Plasmas 6, 3327 (1999).

${ }^{2}$ R. M. Moore, in Handbook of Plasma Physics, Physics of Laser Plasma, edited by A. M. Rubenchik and S. Witkowski (North-Holland, New York, 1991), Vol. 3.

${ }^{3}$ B. Yaakobi, P. Bourke, Y. Conturie, J. Delettrez, J. M. Forsyth, R. D. Frankel, L. M. Goldman, R. L. McCrory, W. Seka, J. M. Soures, A. J. Burek, and R. E. Deslattes, Opt. Commun. 38, 196 (1981).

${ }^{4}$ D. L. Matthews, E. M. Campbell, N. M. Ceglio, G. Hermes, R. Kauffman, L. Koppel, R. Lee, K. Manes, V. Rupert, V. W. Slivinsky, R. Turner, and F. Ze, J. Appl. Phys. 54, 4260 (1983).

${ }^{5}$ D. W. Phillion and C. J. Hailey, Phys. Rev. A 34, 4886 (1986).

${ }^{6}$ G. A. Kyrala, R. D. Fulton, E. K. Wahlin, L. A. Jones, G. T. Schappert, J. A. Cobble, and A. J. Taylor, Appl. Phys. Lett. 60, 2195 (1992).

${ }^{7}$ G. J. Tallents, M. H. Key, A. Ridgeley, W. Shaikh, C. L. S. Lewis, D. O’Neill, S. J. Davidson, N. J. Freeman, and D. Perkins, J. Quant. Spectrosc. Radiat. Transf. 43, 53 (1990).

${ }^{8}$ J. C. Gauthier, S. Bastiani, P. Audebert, J. P. Geindre, K. Neuman, T. Donnelly, M. Hoffer, R. W. Falcone, R. Sheperd, D. Price, and B. White, Proc. SPIE 2523, 242 (1995).

${ }^{9}$ R. Kauffman, in Handbook of Plasma Physics, Physics of Laser Plasma, edited by A. M. Rubenchik and S. Witkowski (North-Holland, New York, 1991), Vol. 3.

${ }^{10}$ S. G. Glendinning, P. Amendt, K. S. Budil, B. A. Hammel, D. H. Kalantar, M. H. Key, O. L. Landen, B. A. Remington, and D. E. Desenne, Proc. SPIE 2523, 29 (1995)

${ }^{11}$ J. Dunn, B. K. F. Young, A. L. Osterheld, M. E. Foord, R. S. Walling, R. E. Stewart, and A. Ya. Faenov, Proc. SPIE 2523, 254 (1995).

${ }^{12}$ T. R. Boehly, D. L. Brown, R. S. Craxton, R. L. Keck, J. P. Knauer, J. H. Kelly, T. J. Kessler, S. A. Kumpan, S. J. Loucks, S. A. Letzring, F. J. Marshall, R. L. McCrory, S. F. B. Morse, W. Seka, J. M. Sources, and C. P. Verdon, Opt. Commun. 133, 496 (1997).

${ }^{13}$ L. N. Koppel and J. D. Eckels, UCRL-79781 (1977).

${ }^{14}$ B. L. Henke, J. Y. Uejio, G. F. Stone, C. H. Dittmore, and F. G. Fujiwara, J. Opt. Soc. Am. B 3, 1540 (1986).

${ }^{15}$ A. B. Bullock et al., Rev. Sci. Instrum. (these proceedings).

${ }^{16}$ G. A. Kyrala and J. Workman, Rev. Sci. Instrum. (these proceedings). 